\title{
Research on Resource Scheduling in Cloud Computing Based on Firefly Genetic Algorithm
}

\author{
Jiyu Chen \\ (Zhejiang Industry Polytechnic College, Shaoxing 312000, China) \\ zjsxcjy@sina.com
}

\begin{abstract}
Cloud computing resource scheduling efficiency is the direction of the current research. In this paper, we first set up a resource scheduling model in cloud computing environment, and put the individual and the cloud computing node resources in the firefly algorithm. Secondly, in individual initialization algorithm introduced genetic algorithm optimize the initial solution, on location algorithm update setting sensory threshold used to adjust the individual probability of selecting the optimal path. Finally, the improvement of the volatile factor makes the value of the fluorescence to be updated. Simulation results show that the proposed algorithm can effectively improve the performance of resource scheduling in cloud computing, shorten the time to complete the task, and improve the overall processing capacity of the system.
\end{abstract}

Keywords: Cloud Computing; Firefly Algorithm; Genetic Algorithm

\section{Introduction}

In recent years, cloud computing is a kind of integrated technology, which is developed on the technology of distributed computing, virtualization technology, grid computing and Web services [1-2]. Cloud computing through the Internet will be in the resources of each node in the network to share. But how to maximize the reasonable utilization of resource scheduling is a hot direction of cloud computing research.

At present, the domestic and foreign scholars have carried out a lot of research work on resource scheduling in cloud computing environment. In literature [3], a modified particle swarm optimization algorithm based on improved particle swarm optimization algorithm is proposed to find the optimal solution of the cloud resource scheduling problem with PSO fast convergence speed, and according to the fitness value of each particle, the speed weight of each particle is changed to improve the global optimization ability and convergence ability. The simulation results show that the average completion time of the task is reduced and the efficiency of task processing is improved; The literature [4] proposed the integration of virtual resources, cloud services, based on the fuzzy set theory is introduced into the cloud resource selection based on quality of service (QoS), and achieved a certain effect; In literature [5], the information entropy theory is used to maintain the non-dominated solution set, in order to keep the diversity and distribution of the solution; Based on the fast convergence of the Sigma method, the chaos disturbance mechanism is introduced to improve the population diversity and the global optimization ability. In literature [6], proposes a cloud computing load balancing bidirectional ant colony optimization algorithm for resource scheduling. The algorithm takes into account the load and computing power of each virtual machine, and introduces the ant to move forward and backward in the cloud environment; Finally, through the simulation experiment on CloudSim platform, the results show that the total task completion time of the algorithm is shorter, and it has a better ability to search and load balance, and it is an effective resource scheduling algorithm. In literature [7], a bat algorithm based on membrane calculation is put 
forward. The internal decomposition of the membrane system is the main membrane and the auxiliary membrane, the individual local optimization of the bat is carried out in the auxiliary film, and the optimized individual is transferred to the main membrane to achieve global optimization. The experimental results show that the algorithm improves the system processing time and efficiency under the cloud computing environment, which makes the resource allocation more reasonable in the cloud computing environment. In literature [8], an adjustment method of elastic resources for Cloud Computing Based on application performance is proposed. The method uses the automatic scaling algorithm to adjust the load demand fluctuation on the vertical level, in order to realize the demand of the service level of the application, and optimize the resource utilization of the cloud. In literature [9], a cloud computing task resource allocation model based on Q learning and bi directional ACO algorithm is proposed; First of all, based on the master slave structure of the scheduling model, and considering the completion time of task computing, network bandwidth and delay and other factors, the resource allocation objective function is designed. Then, the initial allocation method of cloud computing resources based on Q learning is designed to initialize the Q value of the node in the network. Finally, the ACO algorithm is designed to achieve the ultimate allocation of task resources. In literature [10], a low load and low cost resource allocation strategy is proposed to realize the system load balancing. Experimental results show that the proposed method can effectively improve the utilization of resources under the condition of satisfying the QoS constraint.

In this paper, the genetic algorithm is introduced to improve the genetic algorithm based on the problem of the resource scheduling, which makes the improved algorithm to improve the allocation of resources in the process of parallel tasks. Through experiments, it is proved that this algorithm has a certain advantage in the cloud computing resource allocation.

\section{Resource Allocation Model in Cloud Computing Environment}

Resource scheduling in cloud resources is represented by the following model:

$$
\left\{\begin{array}{c}
\left.\max \sum_{i} f_{i} y_{i} \quad\right) \\
\text { s.t. } \sum_{j} x_{i j}=y_{i} \\
\sum_{i} x_{i j} \leq C_{j} \\
\frac{I_{i}}{D_{i}} \leq y_{i} \\
x_{i j} \geq 0 \\
\min \sum_{i, j} C(i, j) \\
\max \sum_{i, j} E(i, j) \\
\min \sum_{i, j} S(i, j)
\end{array}\right.
$$

(1)

In formula (1), $C_{j}$ represents the maximum computational processing power of a virtual machine $\mathrm{J}, I_{i}$ indicates the number of machine instructions contained in the job $i, D_{i}$ represents the latest completion time of the task, and $y_{i}$ represents the sum of the resources allocated to the task $i$, expressed as $y_{i}=\sum_{j} x_{i j}$, Among them, $\sum_{j} x_{i j}$ is the sum of the computing resources occupied by the virtual machine $j . f_{i}\left(y_{i}\right)$ is expressed as the efficiency function of the objective function. According to the requirement of resource scheduling of cloud computing, cloud computing resource 
scheduling is the maximum of $\max \sum_{i} f_{i}\left(y_{i}\right)$

\section{Fundamental Algorithm}

\subsection{Artificial Firefly Algorithm}

Hypothesis of the firefly group $N$, the $i$ firefly where $\left(x_{i}, y_{i}\right)$, corresponding to the $i$ fireflies of the objective function is $f\left(x_{i}, y_{i}\right)$, the $i$ firefly fluorescent pigment value for $T_{i}, x_{j}(t)$ denotes the position of the first generation $t$ of the $j$ a firefly, $l_{j}(t)$ denotes the generation $t$ of the $j$ a luciferin value, firefly's field of view is updated as follows:

$R_{d}^{j}(t)=\max \left\{0, R_{d}^{j-1}(t)+\beta\left(\left|N_{i}(t)\right|\right)\right\}$

(2)

$N_{i}(t)=\left\{\left\|x_{j}(t-1)-x_{i}(t-1)\right\|<R_{d}^{i}\right\}$

(3)

The update formula of the firefly individual position:

$x_{i}(t)=x_{i}(t-1)+\frac{x_{i}(t-1)-x_{i-1}(t-1)}{\left\|x_{i}(t-1)-x_{i-1}(t-1)\right\|}$

(4)

Formula of fluorescein value:

$l_{i}(t+1)=(1-\rho) l_{i}(t)+\lambda J\left(x_{i}(t+1)\right)$

(5)

\subsection{Genetic Algorithm}

The algorithm is a random probability search algorithm which simulates the genetic selection and natural selection. The algorithm consists of 4 steps:

(1) Data encoding program identified, randomly generated an individual;

(2) The fitness value of individual evaluation is given, and the individual's evaluation is to calculate the length of the path for each individual, and the length as an individual fitness function, which is expressed as follows:

$f(x)=\sum_{i=1}^{n-1}$ length $(i, j)+d(1, n)$

(6)

In the formula (6), length $(i, j)$ represents the distance between the two individuals $i$ and $j$. The smaller the degree of adaptation, the shorter the individual indicates that the individual is better.

(3) Judge whether the algorithm meets the conditions of convergence, if the output search results are satisfied, otherwise continue to perform

(4) According to the crossover probability and mutation probability, the crossover operation is performed.

\section{Application of Firefly Genetic Algorithm in Cloud Computing Resources}

\subsection{Optimization of Individual Initial Solution}

Genetic algorithm is adopted to initial solution of the original firefly, which makes 
the efficiency of the firefly algorithm improved significantly. Set firefly algorithm the population size $M$, all fireflies individual is divided into two populations, respectively, the parent populations and sub populations. Where the parent population is $Z=\left\{\alpha_{1}, \alpha_{2}, \ldots a_{M}\right\}$, the fitness function of each individual is set as $f\left(\alpha_{i}\right)$, subgroup $X=\left\{\delta_{1}, \delta_{2}, \ldots \delta_{M}\right\}$, all of the parent population in accordance with the order from the big to small, the sort after the population is $Z^{\prime}=\left\{\alpha_{1}^{\prime}, \alpha_{2}^{\prime}, \ldots a_{M}^{\prime}\right\}$, the fitness value from the size to the small $f\left(\alpha_{1}^{\prime}\right)>f\left(\alpha_{2}^{\prime}\right)>\ldots \ldots . f\left(a_{M}^{\prime}\right)$. In order to calculate the probability of each individual $\sum_{i=1}^{M} f\left(\alpha_{i}^{\prime}\right)$, the probability of each individual cumulative probability is $P(i)=\frac{f\left(\alpha_{i}^{\prime}\right)}{\sum_{i=1}^{M} f\left(\alpha_{i}^{\prime}\right)}$, using the roulette strategy to generate $M$ random number $R$, if $R \leq Q_{i}$, then select the chromosome $b_{1}$, otherwise, we select the $b_{1}$ chromosome. Therefore, the selection of a $M_{i}$ for the $b_{\min (i 1, i 2, \ldots i n)}$, the $M_{i}$ and subgroup $X$ to be combined, making $X(t)=X(t-1) \cup M_{i}$. Then these individuals are operated by crossover, mutation and so on, and the final solution is obtained as the initial solution of the firefly algorithm.

\subsection{The Threshold Setting - Individual Choice}

Firefly individuals in the forward direction selection strategy is according to fluorescein value to judge, which easily lead to in a certain direction, prone to local optima, leading to the subsequent firefly individual existence with larger probability centralized selection in the forward position of the local optimization, in order to avoid the occurrence of such a situation, set a threshold of $\gamma$, the setting value is smaller than the threshold value of, individual fireflies can ignore a value to the advancing direction of fluorescein on the path, so you can continue to look for. On the contrary, the firefly individual choice to select the direction of the advance of the fluorescein.

Therefore, the first $k$ firefly individual according to the following probability from the state $i$ to state $j$ to carry on the transformation, in which allowed $_{k}$ is the state list.

$j=\max \left\{\tau_{i}^{\alpha}, \eta_{i}^{\beta}\right\}, s \in$ allowed $_{k}$ if $\lambda<\gamma$

\subsection{Improvement of $\rho$}

In formula (5), due to the role of factor $\rho$ in the update formula, the value of fluorescein on the search path is gradually reduced until it is not selected. At the same time, the value of the factor $\rho$ on the search path gradually becomes larger, the information content of the effective solution is gradually increased, leading to the reduction of the individual solution of the global search optimization ability. Therefore, it is important to deal with the factor $\rho$. In this paper, the adaptive factor $\eta$ is used to change the value of $\rho$, the initial value of $\rho$ is 0.99 , the minimum is $\rho_{\min }$, and the number of cycles is gradually decreasing;

$$
\rho(t+1)=\left\{\begin{array}{l}
0.99-\eta \llbracket \text { rand }() / 100 \sqsubset \text { time } \rho(t)>\rho_{\text {min }} \\
\rho_{\text {min }}
\end{array}\right.
$$


In (8) the $\operatorname{rand}()$ is a random function, the $\eta$ is the adaptive factor, and the time is the number of cycles, which can guarantee the global search ability of the algorithm in a certain degree of search.

\subsection{Algorithm Step Description}

The solution procedure is shown below, as shown in Figure 2.

Step1: To initialize the various parameters of the firefly algorithm, set the number of $N u m$, the number of iterations of the algorithm is $N$, the maximum number of $N$, to meet the $N<\max$, initialization of the individual position of the firefly, the appropriate value of the;

Step2: According to the characteristics of cloud computing resource allocation, the resources of cloud computing nodes and the individual location of the firefly are one corresponding;

Step3: According to the 3.1 description of the firefly algorithm to initialize the individual;

Step4: According to the formula (2) and (3) calculate the position of a single firefly and the value of the fluorescein to find the position of the respective node and the value of the fluorescein.

Step5: According to the formula (4) (5) to calculate the best location of the firefly, and then according to the formula (7) (8) to update the location of the firefly Gauss mutation, in the current position of the individual to get a new position $x_{\text {new }}(t), x_{i}(t)$.

Step6: Calculate each firefly $x_{\text {new }}(t)$, if $x_{\text {new }}(t)>x_{i}(t)$, then $i=n e w$, then update.

Step7: If the number of iterations is less than max , go to Step8.

Step8: If the termination condition is satisfied, the optimization process will be over, otherwise the Step4 will continue to optimize.

Step9: According to the position of the optimal individual in the firefly, the resource allocation scheme for cloud computing. 


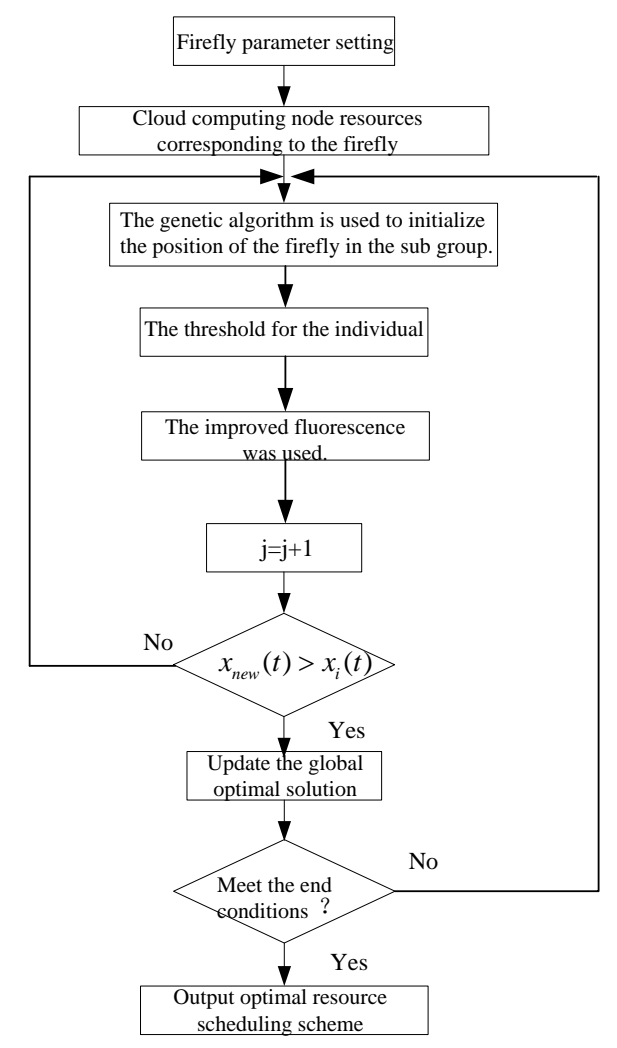

Figure 1. The Cloud Computing Resource Scheduling Based on Firefly Genetic Algorithm

\section{Algorithm Simulation}

In order to further verify the superiority of the algorithm in cloud computing resource scheduling, this paper from 2 aspects: (1) compared with the representative reference published in recent years; (2) compared with the classic intelligent algorithms. The platform of CloudSim[14] tests were carried out, select the CPU core I3 and 4GDDR3, Windows XP, the simulation software using B was simulated.

\subsection{Compared with the Recent Literature Algorithm}

Set the virtual task for 400 , the virtual node for 100 , set the number of iterations is 300 , the algorithm and literature [3] and literature [10] algorithm in the cloud computing model for different tasks.

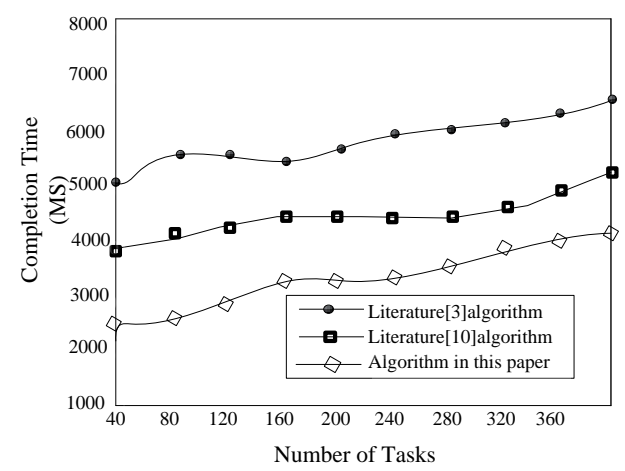

Figure 2. Comparison of 3 Resource Load Algorithms Task Completion Time 


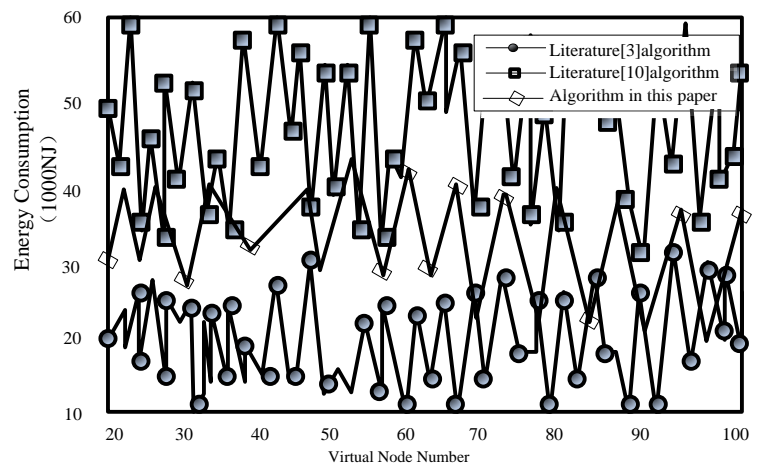
Figure 3. Comparison of Energy Consumption Time of 3 Resource
Load Algorithms

\subsection{Compared with the Classical Intelligent Algorithms}

In this paper, we select the three different kinds of virtual tasks under the cloud computing, and compare the performance of the algorithm. The number of tasks is 100 thousand and the number of tasks is 1 million as the research object.

(1) Performance Comparison of 100 Tthousand Algorithms for Virtual Tasks

The use of genetic algorithm, firefly algorithm, the task is 100 thousand and the number of resources for the allocation of 1000, compared with the results shown in figure 4. From Figure 4, we can find that the number of tasks began to distinguish, and the algorithm and the firefly algorithm in the initial stage of the difference is not large, with the increasing number of tasks, the three algorithms presented a relatively large difference, the proposed algorithm is suitable for the cloud computing conditions of virtual task scheduling problem.

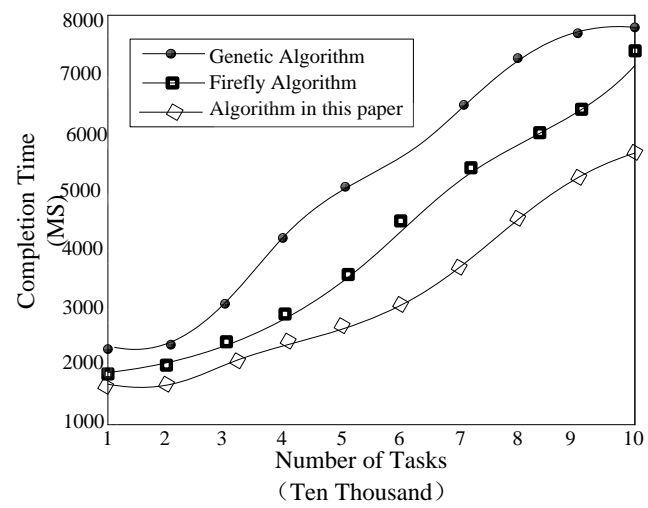

Figure 4. Performance Comparison of 100 Thousand Algorithms for Virtual Tasks

(3) Performance Comparison of 1 Million Algorithms for Virtual Tasks

The use of genetic algorithm, firefly algorithm, the task is 1 million and the number of resources for the allocation of 1000, compared with the results shown in figure 5. From Figure5 shows that, with the increase of the number of virtual task, when the number of tasks to reach 50 million, the algorithm of the task completion time is much less than the other, mainly because the algorithm for the initial individuals and the threshold setting and the $\rho$ was improved in the paper. The algorithm in this paper is suitable for the very large resource scheduling problem in the very suitable for cloud computing, and the effect is more obvious. 


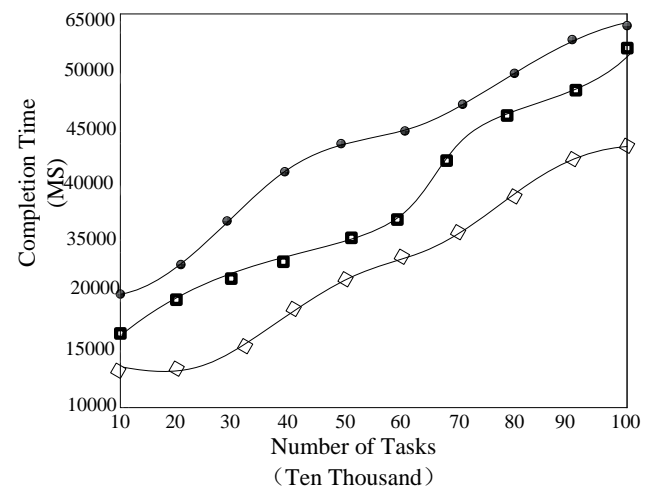

Figure 5. Performance Comparison of 1 Million Algorithms for Virtual Tasks

\section{Conclusion}

Through the establishment of a cloud computing environment of resource scheduling model, individuals of firefly algorithm and cloud computing resource nodes corresponding, secondly in individual initialization algorithm into genetic algorithm optimize the initial solution, the position of the algorithm in the update set sensory threshold used to adjust a selection probability of the optimal path. Finally, the improvement of the volatile factor makes the value of the fluorescence to be updated. The experimental results show that the proposed algorithm can effectively meet the cloud computing resource allocation model, which provides a reference value for the distribution of cloud resources.

\section{References}

[1] LI Qiao.Research survey of Cloud computer[j].Computer Science, 2011,38(4):32-36

[2] Ge xin.Scheduling Problem Research Based on the Use of Cloud Computing for Cluster Expansion[D].HeFei:Universtiy of Science and Technology of China.2011

[3] CAI Qi;SHAN Donghong;ZHAO Weiting.Resource scheduling in cloud computer based on improved particle swarm optimization algorithm[j].Journal of Liaoning Technical University(Natural Science), 2015,35(1):93-96

[4] LIU Na-na;WEI Wang-yang;WU Qing-tao.The Way of Cloud service Selection Based on Fuzzy QoS[j],Microelectronics \& Computer, 2015,32(8):41-44

[5] ZHANG Heng-wei;HAN Ji-hong;WEI Bo.Research on Cloud Resource Scheduling Method Based on Map-Reduce[j].Computer Science, 2015,42(8):118-121

[6] Wang Changfang;Xu Wenzhong.A Bidirectional Ant Colony Optimization Alporithm for Cloud Computer Resource Scheduling[j].Computer Measurement \& Control, 2015,23(8):2861-2863

[7] NING Bin;GU Qiong;WU Zhao.Bats algorithm research in cloud computer resource scheduling based on membrane computing[j].Application Research of Computers, 2015,32(3):830-833

[8] Shen Jing;Wu Chenguang;Hao Yang.Elastic resource adjustment method for cloud computing data center[j].Journal of Nanjing University of Science and Technology, 2015,39(1):89-93

[9] Sun Hua;Zhu Jinxin.Design of Task -Resource Allocation Model Based on Q-Learning and Double Orientation ACO Algorithm for Cloud Computing[j].Computer Measurement \& Control, 2014,22(10):3343-3347

[10] Fang Yiqiu;Zheng Jian;Ge Junwei.A RESOURCE ALLOCATION STRATEGY IN CLOUD ENVIRONMENT BASED ON QoS CONSTRAINT[J].Computer Applications and Software, 2015,32(1):34-38

\section{Author}

Jiyu Chen (1984- ), female, master, lecturer, research direction: information security, wireless sensor and cloud computing 